\title{
Crystal structure of atazanavir, $\mathrm{C}_{38} \mathrm{H}_{52} \mathrm{~N}_{6} \mathrm{O}_{7}$
}

\author{
James A. Kaduk (D), ${ }^{1,2, a)}$ Amy M. Gindhart, ${ }^{3}$ and Thomas N. Blanton (1) ${ }^{3}$ \\ ${ }^{I}$ Illinois Institute of Technology, 3101 S. Dearborn St., Chicago, Illinois 60616, USA \\ ${ }^{2}$ North Central College, 131 S. Loomis St., Naperville, Illinois 60540, USA \\ ${ }^{3}$ ICDD, 12 Campus Blvd., Newtown Square, Pennsylvania 19073-3273, USA
}

(Received 29 October 2019; accepted 26 February 2020)

\begin{abstract}
The crystal structure of atazanavir has been solved and refined using synchrotron X-ray powder diffraction data and optimized using density functional techniques. Atazanavir crystallizes in space group $P 2_{1}$ (\#4) with $a=15.33545(7), b=5.90396(3), c=21.56949(13) \AA, \beta=96.2923(4)^{\circ}, V=$ 1941.134(11) $\AA^{3}$, and $Z=2$. Despite being labeled as "atazanavir sulfate", the commercial reagent sample consisted of atazanavir free base. The structure consists of an array of extended-conformation molecules parallel to the $a c$-plane. Although the atazanavir molecule contains only four classical hydrogen bond donors, hydrogen bonding is, surprisingly, important to the crystal energy. Both intra- and intermolecular hydrogen bonds are significant. The hydroxyl group forms bifurcated intramolecular hydrogen bonds to a carbonyl oxygen atom and an amide nitrogen. Several amide nitrogens act as donors to the hydroxyl group and carbonyl oxygen atoms. An amide nitrogen acts as a donor to another amide nitrogen. Several methyl, methylene, methyne, and phenyl hydrogens participate in hydrogen bonds to carbonyl oxygens, an amide nitrogen, and the pyridine nitrogen. The powder pattern is included in the Powder Diffraction File ${ }^{\mathrm{TM}}$ as entry 00-065-1426. () 2020 International Centre for Diffraction Data. [doi:10.1017/S0885715620000135]
\end{abstract}

Key words: atazanavir, powder diffraction, Rietveld refinement, density functional theory

\section{INTRODUCTION}

Atazanavir, as the sulfate Reyataz ${ }^{\circledR}$, is an HIV-1 protease inhibitor. Atazanavir is typically administered with other antiretrovirals, for example, ritonavir, resulting in a synergistic effect boosting the efficacy of each medication. As the free base, the IUPAC name for atazanavir (CAS Registry number 198904$31-3)$ is methyl $\mathrm{N}-[(2 S)-1-[2-[(2 S, 3 S)-2$-hydroxy-3-[[(2S)-2(methoxycarbonylamino)-3,3-dimethylbutanoyl]amino]-4phenylbutyl]-2-[(4-pyridin-2-ylphenyl)methyl]hydrazinyl]-3,3dimethyl-1-oxobutan-2-yl]carbamate. A two-dimensional molecular diagram for Atazanavir is shown in Figure 1.

A reduced cell search of the Cambridge Structural Database (Groom et al., 2016), increasing the default tolerance from $1.5 \%$ to $2.0 \%$ of the longest cell dimension, yielded 10 hits, but no structure for atazanavir derivatives. A name search on "atazanavir" yielded a single-crystal structure of atazanavir at $150 \mathrm{~K}$ (Patel et al., 2018; CSD Refcode LISTEP; Pfizer; published during the course of this work) and two structures of atazanavir bisulfate (Kim et al., 2011; CSD Refcodes LUQRAS and LUQREW; Bristol-Myers Squibb). The Kim et al. patent application reports the crystal structure and powder data for Form A of atazanavir bisulfate, a process for preparing Form $\mathrm{C}$, and the structure of a triethanol solvate Form E3. Crystallographic data for these forms are also reported in Kim et al. (2005).

This work was carried out as part of a project (Kaduk et al., 2014) to determine the crystal structures of largevolume commercial pharmaceuticals at room temperature for

\footnotetext{
${ }^{\text {a) }}$ Author to whom correspondence should be addressed. Electronic mail: kaduk@polycrystallography.com
}

phase identification applications and include high-quality powder diffraction data for these pharmaceuticals in the Powder Diffraction File (Gates-Rector and Blanton, 2019).

\section{EXPERIMENTAL}

The sample labeled as "atazanavir sulfate" was a commercial reagent, purchased from Arking Pharma Scientific, Inc. (Lot \#Arki-24306), and was used as-received. The white powder was packed into a 1.5-mm diameter Kapton capillary and rotated during the measurement at $\sim 50 \mathrm{~Hz}$. The powder pattern was measured at $295 \mathrm{~K}$ at beamline 11-BM (Lee et al., 2008; Wang et al., 2008) of the Advanced Photon Source at Argonne National Laboratory using a wavelength of $0.413891 \AA$ from $0.5^{\circ}$ to $50^{\circ} 2 \theta$ with a step size of $0.001^{\circ}$ and a counting time of $0.1 \mathrm{~s} \mathrm{step}^{-1}$.

The pattern was indexed on a primitive monoclinic unit cell with $a=15.331, b=5.902, c=21.562 \AA, \beta=96.3^{\circ}, V=$ $1939.3 \AA^{3}$, and $Z=2$ using Jade 9.8 (MDI, 2018). The suggested space group was $P 2_{1}$, which was confirmed by the successful solution and refinement of the structure. After many attempts to solve the structure as a sulfate derivative, the $\mathrm{S}$ content was checked by X-ray Photoelectron Spectroscopy (XPS). A surface concentration of a few hundred ppm S was detected, consistent with the formulation as a free base. The unit cell volume of atazanavir bisulfate Form A is 2150(2) $\AA^{3}$. The unit cell volume observed here is $9.3 \%$ smaller, so the compound was unlikely to be a bisulfate.

Once the absence of S was realized, the atazanavir molecule was extracted from the crystal structure of Form E3 of the bisulfate using Materials Studio (Dassault, 2019) and converted to a Fenske-Hall Z-matrix using OpenBabel (O'Boyle 


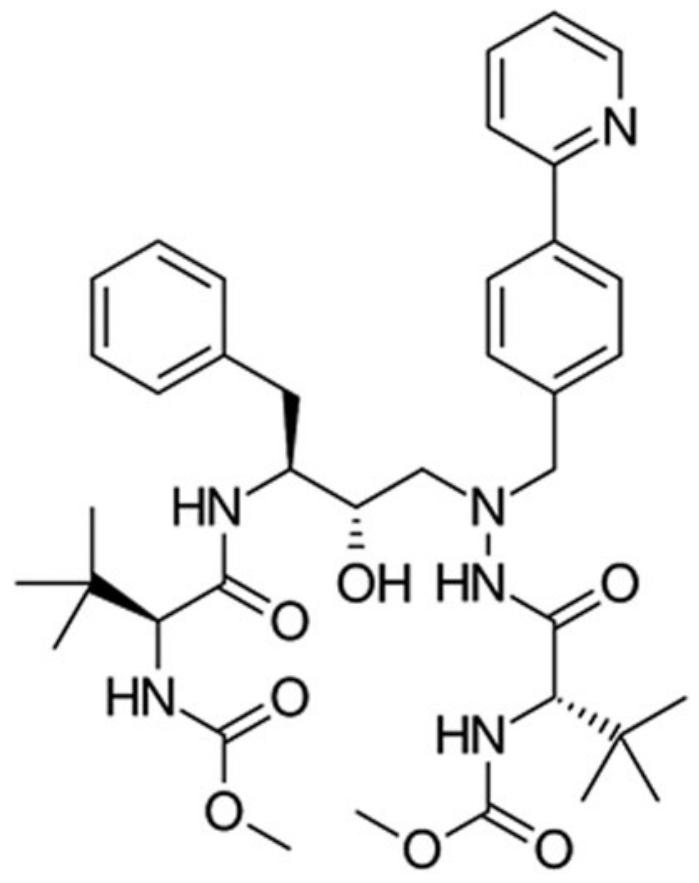

Figure 1. The molecular structure of the atazanavir molecule.

et al., 2011). The structure was solved using FOX (Favre-Nicolin and Černý, 2002).

Initial Rietveld refinement of the structure led to a $w R$ $\sim 17 \%$, an uncomfortably high value. The root-mean-square (rms) Cartesian displacement between the refined structure and a DFT-optimized structure (carried out using the methods described below for the correct structure) was $0.463 \AA$, outside the range for correct structures (van de Streek and Neumann, 2014). Fortunately, by this time, the LISTEP structure had appeared, and we could understand what was wrong with this initial structure from our study. The rms Cartesian displacement between the two structures is $0.942 \AA$ (Figure 2). The differences are spread through the molecules but are largest in the center of the molecule and in the orientation of one of the methyl ester groups on the periphery. The differences are a good reminder that both Rietveld

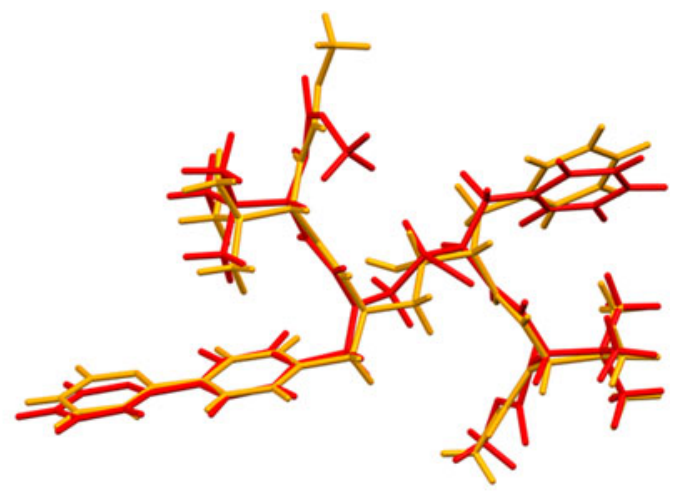

Red $=$ wrong; orange - LISTEP (major); rma delta $=0.942$

Figure 2. (Color online) Comparison of the false-minimum/wrong (red) structure of atazanavir to the correct structure (orange) from the CSD entry LISTEP. The rms Cartesian displacement is $0.942 \AA$. refinement (least squares) and a DFT optimization locate local minima and do not guarantee to find the global optimum structure.

The LISTEP structure has a 68/32\% disorder in one of the side chains (N6-C37-O6-O7-C38). Since an ordered model is necessary for a DFT calculation, only the major orientation of this chain was included in the Rietveld refinement. Refinement was carried out using GSAS-II (Toby and Von Dreele, 2013). Only the $1.5-22.0^{\circ}$ portion of the pattern was included in the refinement $\left(d_{\min }=1.084 \AA\right)$. All non-H bond distances and angles were subjected to restraints, based on a Mercury/Mogul Geometry Check (Bruno et al., 2004; Sykes et al., 2011) of the molecule. The results were exported to a csv file. The Mogul average and standard deviation for each quantity were used as the restraint parameters and were incorporated using the new feature Restraints/Edit Restraints/Add MOGUL Restraints, which reads the bond distance and angle restraints from the csv file. The restraints contributed $2.4 \%$ to the final $\chi^{2}$. The hydrogen atoms were included in calculated positions, which were recalculated during the refinement using Materials Studio (Dassault, 2019). Positions of the active hydrogens were derived by analysis of potential hydrogen bonding patterns. The $U_{\text {iso }}$ of the heavy atoms were fixed at $0.06 \AA^{2}$, and those of the $\mathrm{H}$ atoms at $0.08 \AA^{2}$. The background was modeled using a 3-term shifted Chebyshev polynomial and a 6-term diffuse scattering function to describe the Kapton capillary and any amorphous component.

The final refinement of 180 variables using 20501 observations and 127 restraints yielded the residuals $R \mathrm{wp}=0.09408$ and $\mathrm{GOF}=1.15$. The largest peak $(0.23 \AA$ from C5) and hole (1.12 $\AA$ from C9) in the difference Fourier map were 0.49 and $-0.33(8) e \AA^{-3}$, respectively. The Rietveld plot is included as Figure 3. The largest errors in the fit are in the positions of some of the low-angle peaks and may indicate subtle changes in the beam during the measurement.

A density functional geometry optimization (fixed experimental unit cell) was carried out using VASP (Kresse and Furthmüller, 1996) through the MedeA graphical interface (Materials Design, 2016). The calculation was carried out on $162.4 \mathrm{GHz}$ processors (each with $4 \mathrm{~GB}$ RAM) of a 64-processor HP Proliant DL580 Generation 7 Linux cluster at North Central College. The calculation used the GGA-PBE functional, a plane wave cutoff energy of 400.0 $\mathrm{eV}$, and a $k$-point spacing of $0.5 \AA^{-1}$ leading to a $1 \times 3 \times 1$ mesh, and took $\sim 11 \mathrm{~h}$. A single-point calculation on the VASP-optimized structure was carried out using CRYSTAL14 (Dovesi et al., 2014). The basis sets for the $\mathrm{H}, \mathrm{C}, \mathrm{N}$, and $\mathrm{O}$ atoms were those of Gatti et al. (1994). The calculation was run on eight $2.1 \mathrm{GHz}$ Xeon cores (each with $6 \mathrm{~GB}$ RAM) of a 304-core Dell Linux cluster at IIT, using $8 k$-points and the B3LYP functional, and took $\sim 82 \mathrm{~min}$.

\section{RESULTS AND DISCUSSION}

Despite being labeled as "atazanavir sulfate", this sample consists of atazanavir free base. The refined atom coordinates of atazanavir and the coordinates from the DFT optimization are reported in the CIFs attached in Supplementary Material. As would be expected from a Rietveld-refined structure derived from a single-crystal determination, the rms Cartesian displacement of the non-hydrogen atoms in the 


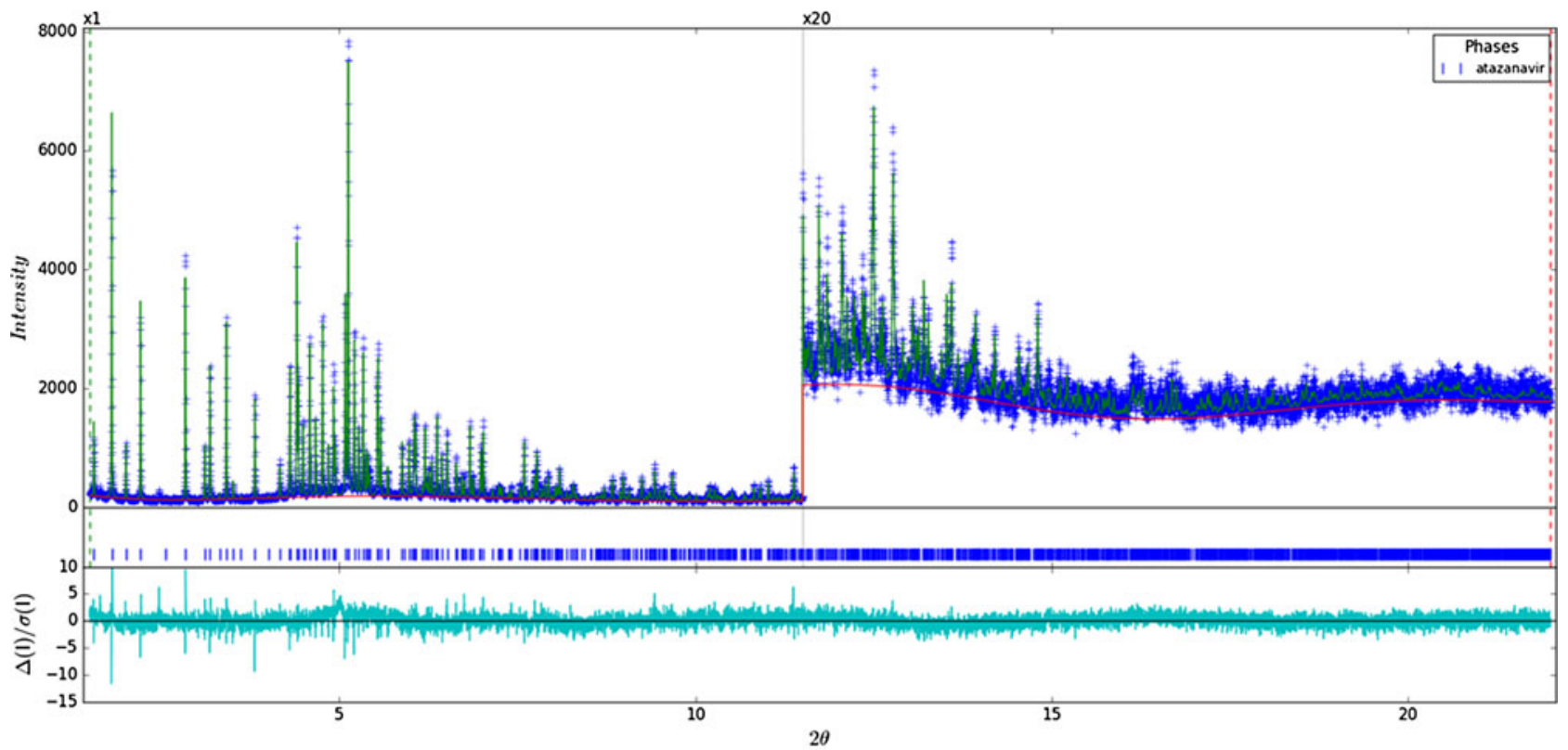

Riet

Figure 3. (Color online) The Rietveld plot for the refinement of atazanavir. The blue crosses represent the observed data points, and the green line is the calculated pattern. The cyan curve is the normalized error plot. The vertical scale has been multiplied by a factor of $20 \times$ for $2 \theta>11.5^{\circ}$.

Rietveld-refined and DFT-optimized structures is $0.077 \AA$ (Figure 4), well within the range expected for correct structures (van de Streek and Neumann, 2014). There is no sign of the disorder noted in the single-crystal structure. Perhaps the samples actually differ, or powder diffraction is not sensitive enough to detect the disorder. This discussion concentrates on the VASP-optimized structure, with the CRYSTAL14 fixed-point calculation. The asymmetric unit (with atom numbering; the LISTEP numbering has been retained) is illustrated in Figure 5, and the crystal structure is presented in Figure 6.

Both the profile residuals $(0.09408$ vs. 0.17246$)$ and the energies $\left(-1.8 \mathrm{kcal} \mathrm{mol}^{-1}\right)$ clearly show that the LISTEP structure is preferred over the initially obtained falseminimum structure (Figure 2). Since the structure solution involves 28 degrees of freedom, we should not be surprised that Monte Carlo simulated annealing might fall into a false minimum. The structure consists of an array of extended-

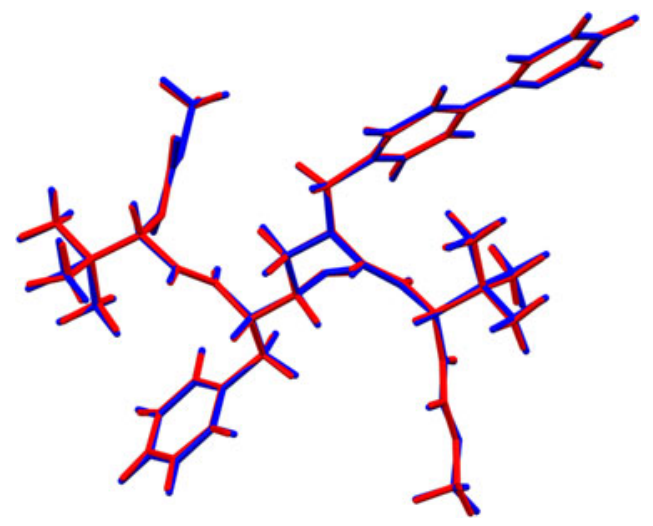

Figure 4. (Color online) Comparison of the Rietveld-refined (red) and VASP-optimized (blue) structures of atazanavir. The rms Cartesian displacement is $0.077 \AA$. conformation molecules parallel to the $a c$-plane (Figure 6). The unit cell expansion between 150 and $295 \mathrm{~K}$ is not especially anisotropic (Table I). Visual inspection suggests that aromatic-aromatic ring interactions and $t$-butyl- $t$-butyl interactions are significant, but this is not supported by the population analysis. All of the bond distances, bond angles, and torsion angles in atazanavir fall within the normal ranges indicated by a Mercury/Mogul Geometry check (Macrae et al., 2008).

Quantum chemical geometry optimization of the atazanavir molecule (DFT/B3LYP/6-31G*/water) using Spartan '18 (Wavefunction, 2018) indicated that the observed solid-state conformation is $9.9 \mathrm{kcal} \mathrm{mol}^{-1}$ higher in energy than the local minimum. The geometry differences are spread throughout the molecule (Figure 7) and lie mainly in the orientations of the arms on the outside of the molecule. Molecular mechanics conformational analysis indicated that the observed conformation is $8.1 \mathrm{kcal} \mathrm{mol}^{-1}$ higher in energy than the global minimum energy conformation (Figure 8). The two conformations differ considerably ( $\mathrm{rms} \Delta=4.091 \AA$ ), suggesting that intermolecular interactions play an important role in determining the solid-state conformation.

Analysis of the contributions to the total crystal energy using the Forcite module of Materials Studio (Dassault, 2019) suggests that the intramolecular deformation energy is small, as might be expected for a flexible molecule. The intermolecular energy is dominated by electrostatic attractions, which in this force field-based analysis include cation coordination and hydrogen bonds. The hydrogen bonds are better analyzed using the results of the DFT calculation.

Although the atazanavir molecule contains only four classical hydrogen bond donors, hydrogen bonding is, surprisingly, important to the crystal energy (Table II). Both intraand intermolecular hydrogen bonds are significant. The hydroxyl group $\mathrm{O} 2-\mathrm{H} 48$ forms bifurcated intramolecular hydrogen bonds to the ketone oxygen atom $\mathrm{O} 3$ and the 


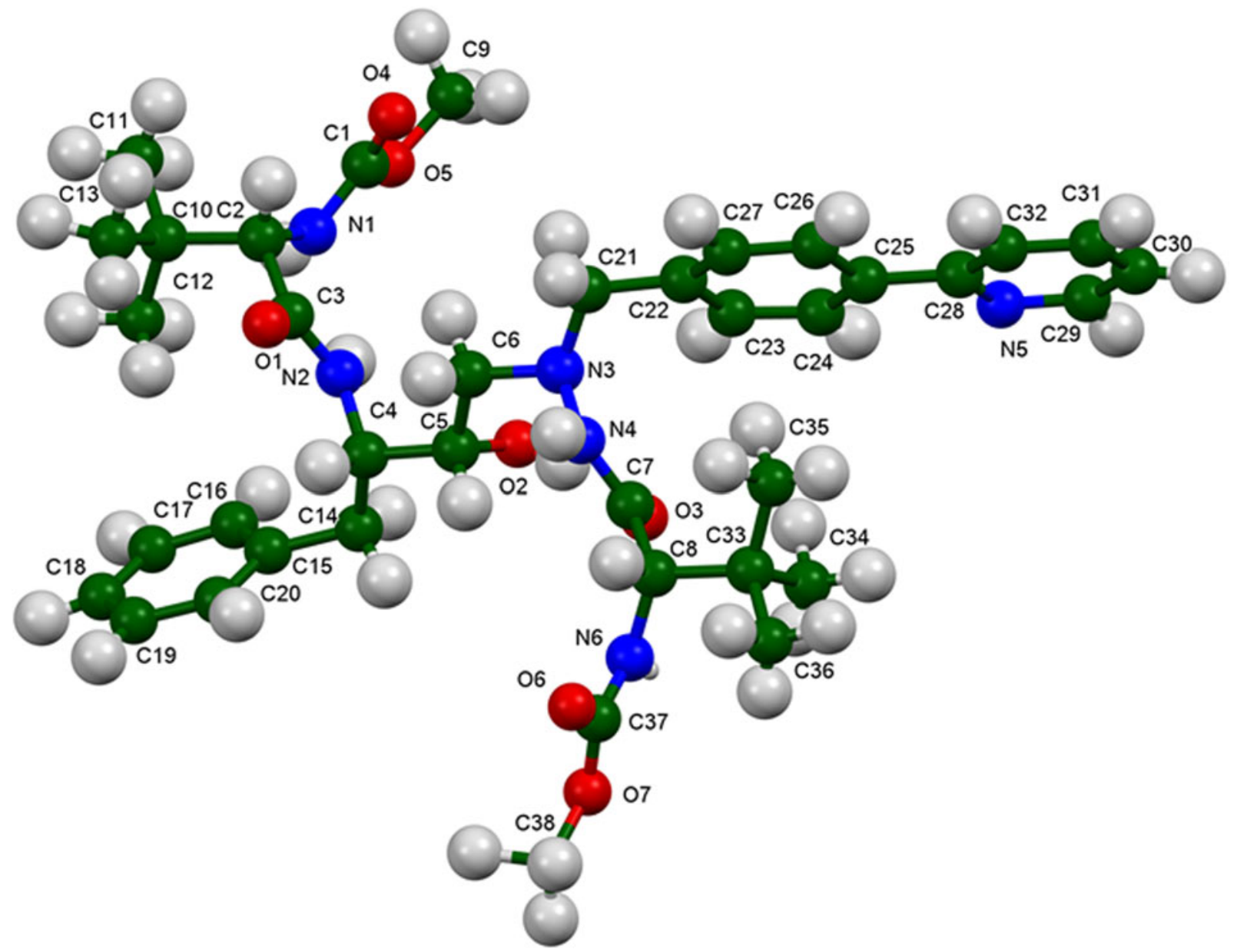

Figure 5. (Color online) The asymmetric unit of atazanavir, with the atom numbering. The atoms are represented by $50 \%$ probability spheroids.

amide nitrogen N3. The energy of the $\mathrm{O}-\mathrm{H} \cdots \mathrm{O}$ hydrogen bond was calculated from the correlation of Rammohan and Kaduk (2018). The amide nitrogen N4 acts as a donor to the hydroxyl group $\mathrm{O} 2$, and the amide nitrogen $\mathrm{N} 1$ acts as a donor to the carbonyl oxygen $\mathrm{O} 1$. The principal classical hydrogen bonds are illustrated in Figure 9. The energies of the $\mathrm{N}-\mathrm{H}$... O hydrogen bonds were calculated using the correlation of Wheatley and Kaduk (2019). The amide nitrogen N2
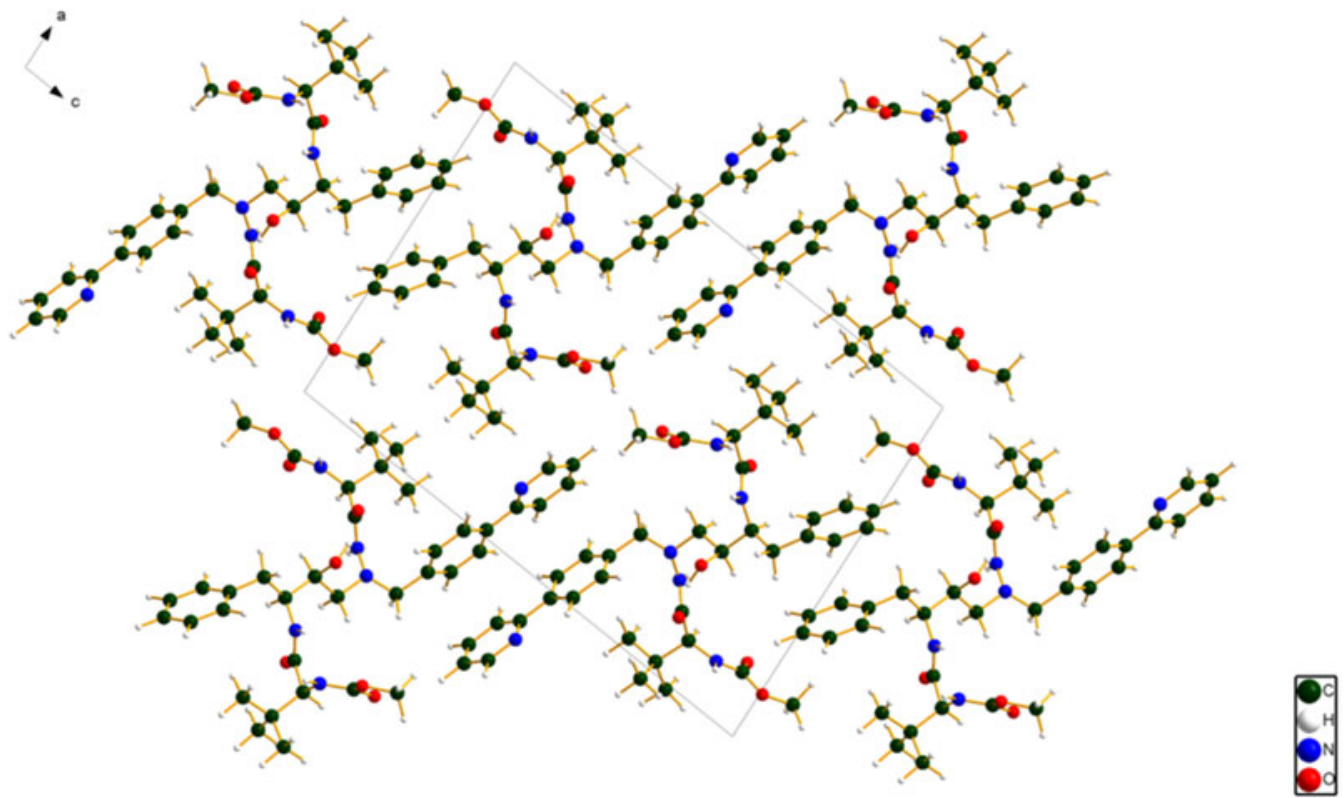

Figure 6. (Color online) The crystal structure of atazanavir, viewed down the $b$-axis. 
TABLE I. Lattice parameters of atazanavir free base. Space group $P 2_{1}$.

\begin{tabular}{llll}
\hline \hline$T(\mathrm{~K})$ & 150 & 295 & Ratio \\
\hline Source & LISTEP & This work & \\
$a(\AA)$ & $15.2650(18)$ & $15.33595(7)$ & 1.0046 \\
$b(\AA)$ & $5.8604(6)$ & $5.90395(3)$ & 1.0074 \\
$c(\AA)$ & $21.365(2)$ & $21.56949(13)$ & 1.0096 \\
$\beta\left({ }^{\circ}\right)$ & $95.966(3)$ & $96.2923(4)$ & \\
$V\left(\AA^{3}\right)$ & 1900.94 & $1941.134(11)$ & 1.0211 \\
\hline
\end{tabular}

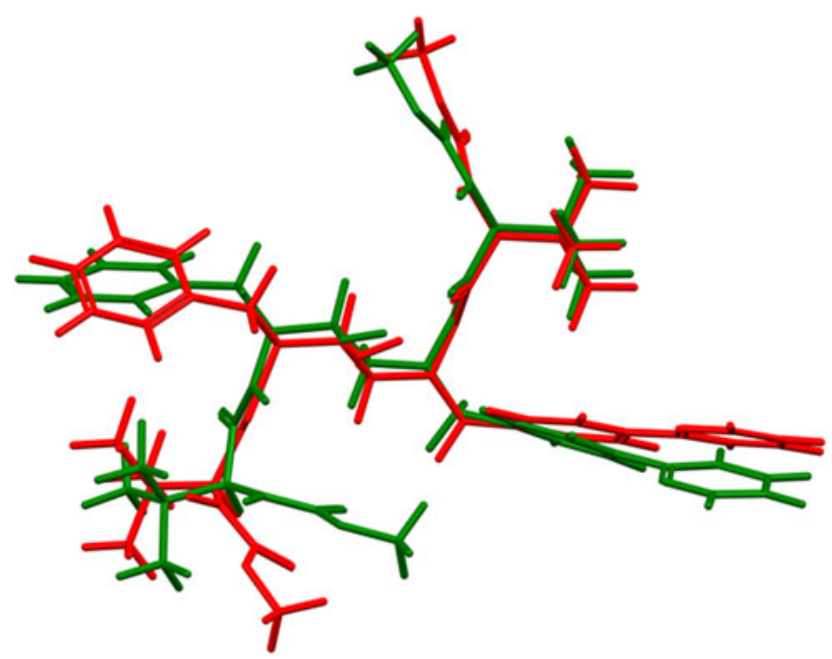

Red = observed solid-state; green = local

minimum isolated molecule; rms delta $=1.097$

Figure 7. (Color online) Comparison of the observed solid-state conformation of atazanavir (red) to the local minimum energy conformation of an isolated molecule (green). The rms Cartesian displacement is $1.097 \AA$.

acts as a donor to the amide nitrogen N1. Several methyl, methylene, methyne, and phenyl hydrogens participate in hydrogen bonds to carbonyl oxygens, the amide nitrogen $\mathrm{N} 2$, and the pyridine nitrogen N5.

The volume enclosed by the Hirshfeld surface (Figure 10; Hirshfeld, 1977; Turner, et al., 2017) is $958.39 \AA^{3}, 98.75 \%$ of one-fourth the unit cell volume. The molecules are, thus, not tightly packed. All of the significant close contacts (red in

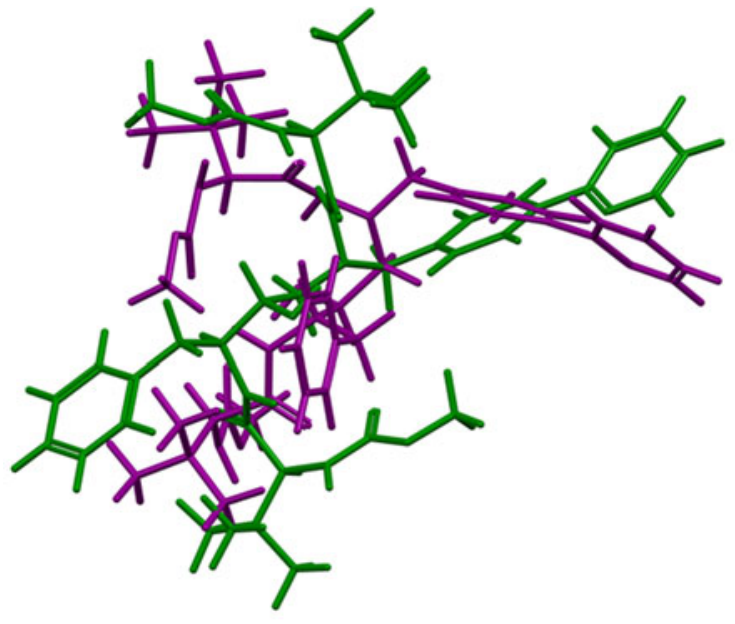

Local $\min =$ green; global $\mathrm{min}=$ purple; $\mathrm{rms}$ delta $=4.091 \mathrm{Ang}$

Figure 8. (Color online) Comparison of the observed solid-state conformation of atazanavir (red) to the global minimum energy conformation of an isolated molecule (purple).

Figure 10) involve the hydrogen bonds. The volume/nonhydrogen atom is $19.0 \AA^{3}$.

The Bravais-Friedel-Donnay-Harker (Bravais, 1866; Friedel, 1907; Donnay and Harker, 1937) morphology suggests that we might expect needle-like morphology for atazanavir, with $\langle 010\rangle$ as the needle axis, as might be expected from the anisotropy of the lattice parameters. A second-order spherical harmonic model for preferred orientation was incorporated into the refinement. The texture index was 1.004, indicating that preferred orientation was not significant in this rotated capillary specimen. The powder pattern of atazanavir from this synchrotron data set is included in the Powder Diffraction File ${ }^{\mathrm{TM}}$ as entry 00-065-1426.

\section{DEPOSITED DATA}

The Crystallographic Information Framework (CIF) files containing the results of the Rietveld refinement (including the raw data) and the DFT geometry optimization were deposited with the ICDD. The data can be requested at info@icdd. com.

TABLE II. Hydrogen bonds (CRYSTAL14) in atazanavir.

\begin{tabular}{|c|c|c|c|c|c|c|}
\hline $\mathrm{H}$ bond & D-H $(\AA)$ & 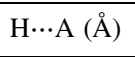 & $\mathrm{D} \cdots \mathrm{A}(\AA)$ & $\mathrm{D}-\mathrm{H} \cdots \mathrm{A}\left({ }^{\circ}\right)$ & Overlap $(e)$ & $E\left(\mathrm{kcal} \mathrm{mol}^{-1}\right)$ \\
\hline $\mathrm{O} 2-\mathrm{H} 48 \cdots \mathrm{O} 3$ & 1.005 & $1.704^{\mathrm{a}}$ & 2.674 & 161.0 & 0.061 & 13.5 \\
\hline $\mathrm{O} 2-\mathrm{H} 48 \cdots \mathrm{N} 3$ & 1.005 & $2.450^{\mathrm{a}}$ & 2.782 & 98.4 & 0.011 & \\
\hline $\mathrm{N} 4-\mathrm{H} 47 \cdots \mathrm{O} 2$ & 1.038 & 1.811 & 2.848 & 177.6 & 0.064 & 5.4 \\
\hline $\mathrm{N} 1-\mathrm{H} 45 \cdots \mathrm{O} 1$ & 1.029 & 2.040 & 3.037 & 162.5 & 0.044 & 4.5 \\
\hline $\mathrm{N} 2-\mathrm{H} 46 \cdots \mathrm{N} 1$ & 1.019 & $2.209^{\mathrm{a}}$ & 2.708 & 108.3 & 0.021 & \\
\hline 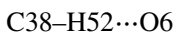 & 1.098 & $2.446^{\mathrm{a}}$ & 2.681 & 90.0 & 0.012 & \\
\hline $\mathrm{C} 35-\mathrm{H} 40 \cdots \mathrm{O} 3$ & 1.099 & 2.422 & 3.458 & 156.4 & 0.012 & \\
\hline $\mathrm{C} 9-\mathrm{H} 7 \cdots \mathrm{O} 4$ & 1.094 & 2.507 & 3.493 & 149.2 & 0.013 & \\
\hline C6-H4 $\cdots \mathrm{N} 2$ & 1.099 & $2.639^{\mathrm{a}}$ & 2.979 & 97.0 & 0.010 & \\
\hline 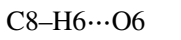 & 1.101 & $2.372^{\mathrm{a}}$ & 2.848 & 104.1 & 0.018 & \\
\hline $\mathrm{C} 2-\mathrm{H} 1 \cdots \mathrm{O} 4$ & 1.102 & $2.392^{\mathrm{a}}$ & 2.858 & 103.6 & 0.015 & \\
\hline $\mathrm{C} 30-\mathrm{H} 33 \cdots \mathrm{O} 4$ & 1.091 & 2.748 & 3.776 & 156.7 & 0.011 & \\
\hline $\mathrm{C} 24-\mathrm{H} 29 \cdots \mathrm{N} 5$ & 1.090 & $2.416^{\mathrm{a}}$ & 2.798 & 98.7 & 0.021 & \\
\hline C18-H23‥O6 & 1.091 & 2.514 & 3.394 & 137.0 & 0.012 & \\
\hline
\end{tabular}

${ }^{\mathrm{a}}$ Intramolecular. 


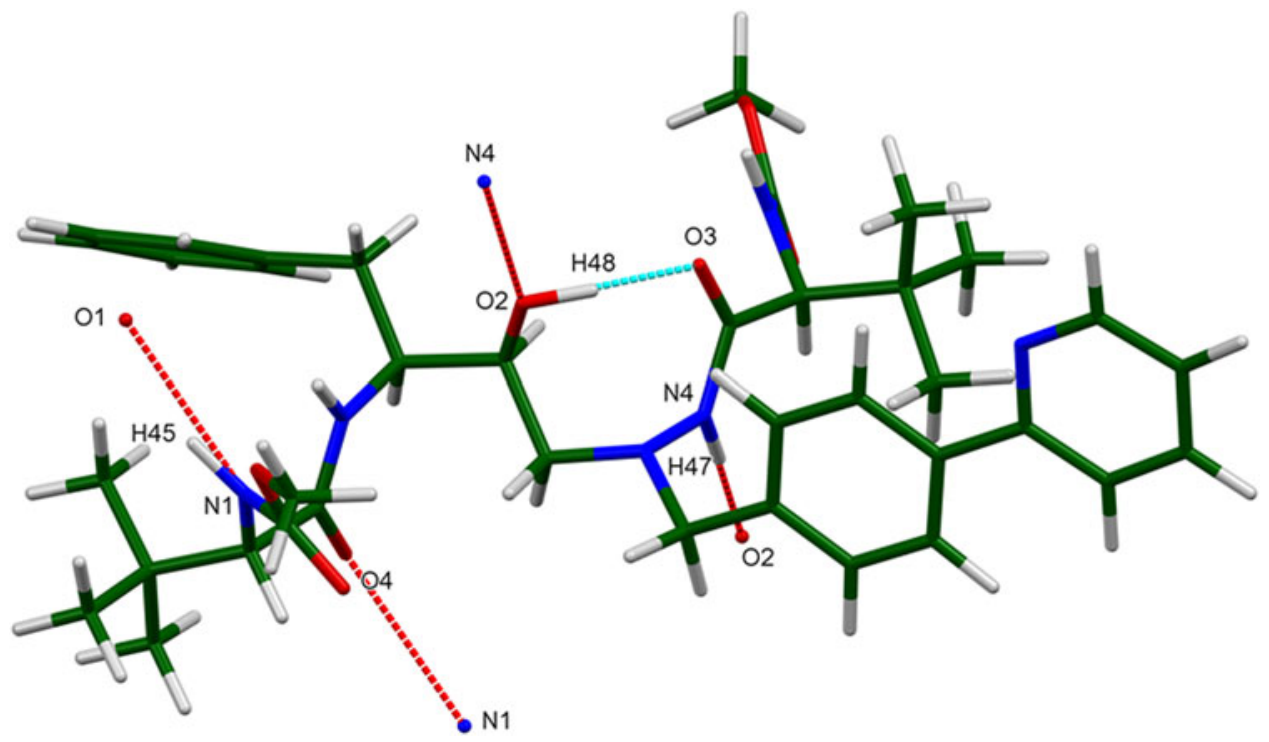

Figure 9. (Color online) The principal classical hydrogen bonds in atazanavir.

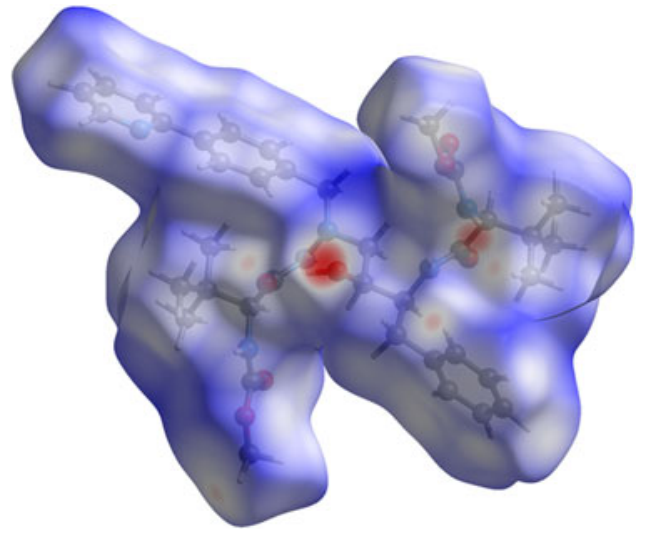

Figure 10. (Color online) The Hirshfeld surface of atazanavir. Intermolecular contacts longer than the sums of the van der Waals radii are colored blue, and contacts shorter than the sums of the radii are colored red. Contacts equal to the sums of radii are white.

\section{ACKNOWLEDGEMENTS}

The use of the Advanced Photon Source at Argonne National Laboratory was supported by the U.S. Department of Energy, Office of Science, Office of Basic Energy Sciences, under Contract No. DE-AC02-06CH11357. This work was partially supported by the International Centre for Diffraction Data. We thank Lynn Ribaud and Saul Lapidus for their assistance in the data collection, Andrey Rogachev for the use of computing resources at IIT, and Gerry W. Zajac of Ineos Technologies for the XPS analysis.

\section{CONFLICTS OF INTEREST}

The authors have no conflicts of interest to declare.

Bravais, A. (1866). Etudes Cristallographiques (Gauthier Villars, Paris). Bruno, I. J., Cole, J. C., Kessler, M., Luo, J., Motherwell, W. D. S., Purkis, L. H., Smith, B. R., Taylor, R., Cooper, R. I., Harris, S. E., and Orpen, A. G. (2004). "Retrieval of crystallographically-derived molecular geometry information,” J. Chem. Inf. Sci. 44, 2133-2144.

Dassault Systèmes (2019). Materials Studio 2019 (BIOVIA, San Diego, CA).
Donnay, J. D. H. and Harker, D. (1937). "A new law of crystal morphology extending the law of Bravais," Am. Mineral. 22, 446-447.

Dovesi, R., Orlando, R., Erba, A., Zicovich-Wilson, C. M., Civalleri, B., Casassa, S., Maschio, L., Ferrabone, M., De La Pierre, M., D-Arco, P., Noël, Y., Causà, M., and Kirtman, B. (2014). "CRYSTAL14: a program for the ab initio investigation of crystalline solids," Int. J. Quantum Chem. 114, 1287-1317.

Favre-Nicolin, V. and Černý, R. (2002). "FOX, 'free objects for crystallography': a modular approach to ab initio structure determination from powder diffraction," J. Appl. Crystallogr. 35, 734-743.

Friedel, G. (1907). "Etudes sur la loi de Bravais," Bull. Soc. Fr. Mineral. 30, 326-455.

Gates-Rector, S. and Blanton, T. (2019). "The powder diffraction file: a quality materials characterization database," Powd. Diffr. 39(4), 352-360.

Gatti, C., Saunders, V. R., and Roetti, C. (1994). "Crystal-field effects on the topological properties of the electron-density in molecular crystals - the case of urea," J. Chem. Phys. 101, 10686-10696.

Groom, C. R., Bruno, I. J., Lightfoot, M. P., and Ward, S. C. (2016). "The Cambridge structural database," Acta Crystallogr. B Struct. Sci. Cryst. Eng. Mater. 72, 171-179.

Hirshfeld, F. L. (1977). "Bonded-atom fragments for describing molecular charge densities," Theor. Chem. Acta 44, 129-138.

Kaduk, J. A., Crowder, C. E., Zhong, K., Fawcett, T. G., and Suchomel, M. R. (2014). "Crystal structure of atomoxetine hydrochloride (Strattera), $\mathrm{C}_{17} \mathrm{H}_{22} \mathrm{NOCl}$," Powd. Diffr. 29(3), 269-273.

Kim, S., Lotz, B. T., Malley, M. F., Gougoutas, J. Z., Davidovich, M., and Srivastava, S. (2005). "Process for preparing atazanavir bisulfate and novel forms," U.S. Patent Application 2005/0256202.

Kim, S., Lotz, B. T., Malley, M. F., Gougoutas, J. Z., Davidovich, M., and Srivastava, S. (2011). "Process for preparing atazanavir bisulfate and novel forms," U.S. Patent Application 2011/0124689 A1.

Kresse, G. and Furthmüller, J. (1996). "Efficiency of ab-initio total energy calculations for metals and semiconductors using a plane-wave basis set," Comput. Mater. Sci. 6, 15-50.

Lee, P. L., Shu, D., Ramanathan, M., Preissner, C., Wang, J., Beno, M. A., Von Dreele, R. B., Ribaud, L., Kurtz, C., Antao, S. M., Jiao, X., and Toby, B. H. (2008). "A twelve-analyzer detector system for highresolution powder diffraction,” J. Synch. Rad. 15(5), 427-432.

Macrae, C. F., Bruno, I. J., Chisholm, J. A., Edington, P. R., McCabe, P., Pidcock, E., Rodriguez-Monge, L., Taylor, R., van de Streek, J., and Wood, P. A. (2008). "Mercury CSD 2.0 - new features for the visualization and investigation of crystal structures," J. Appl. Crystallogr. 41, 466-470.

MDI (2018). Jade 9.8 (Materials Data Inc., Livermore, CA).

O’Boyle, N., Banck, M., James, C. A., Morley, C., Vandermeersch, T., and Hutchison, G. R. (2011). "Open babel: an open chemical toolbox," J. Chem. Informatics 3, 33 . 
Patel, M. A., Luthra, S., Shamblin, S. L., Arora, K. K., Krzyzaniak, J. F., and Taylor, L. S. (2018). "Assessing the risk of salt disproportionation using crystal structure and surface topography analysis," Cryst. Growth Des. 18, 7027-7040.

Rammohan, A. and Kaduk, J. A. (2018). "Crystal structures of alkali metal (Group 1) citrate salts,” Acta Crystallogr. B Cryst. Eng. Mater. 74, 239-252.

Sykes, R. A., McCabe, P., Allen, F. H., Battle, G. M., Bruno, I. J., and Wood, P. A. (2011). "New software for statistical analysis of Cambridge Structural Database data,” J. Appl. Crystallogr. 44, 882-886.

Toby, B. H. and Von Dreele, R. B. (2013). "GSAS II: the genesis of a modern open source all purpose crystallography software package," J. Appl. Crystallogr. 46, 544-549.

Turner, M. J., McKinnon, J. J., Wolff, S. K., Grimwood, D. J., Spackman, P. R., Jayatilaka, D., and Spackman, M. A. (2017).
CrystalExplorer17 (University of Western Australia). Available at: http://hirshfeldsurface.net.

van de Streek, J., and Neumann, M. A. (2014). "Validation of molecular crystal structures from powder diffraction data with dispersion-corrected density functional theory (DFT-D)," Acta Crystallogr. B Struct. Sci. Cryst. Eng. Mater. 70(6), 1020-1032.

Wang, J., Toby, B. H., Lee, P. L., Ribaud, L., Antao, S. M., Kurtz, C., Ramanathan, M., Von Dreele, R. B., and Beno, M. A. (2008). "A dedicated powder diffraction beamline at the Advanced Photon Source: commissioning and early operational results," Rev. Sci. Instrum. 79, 085105.

Wavefunction, Inc (2018). Spartan '18 Version 1.2.0 (Wavefunction Inc., Irvine, CA).

Wheatley, A. M., and Kaduk, J. A. (2019). "Crystal structures of ammonium citrates," Powd. Diffr. 34, 35-43. 